\title{
Effect of Home-Based Pulmonary Rehabilitation on Functional Capacity in People With Idiopathic Pulmonary Fibrosis- A Systematic Review Protocol
}

\section{Revati Amin}

Manipal Academy of Higher Education https://orcid.org/0000-0002-3403-4511

K Vaishali ( $\nabla$ vaishali.kh@manipal.edu )

Manipal Academy of Higher Education https://orcid.org/0000-0001-5746-1682

Arun G Maiya

Manipal Academy of Higher Education

Aswini Kumar Mohapatra

Manipal Academy of Higher Education

Uday Narayan Yadav

University of New South Wales

Shradha S Parsekar

Manipal Academy of Higher Education

\section{Protocol}

Keywords: Functional capacity, Home-based pulmonary rehabilitation, Health-related quality of life, Idiopathic pulmonary fibrosis, Systematic review

Posted Date: February 19th, 2021

DOl: https://doi.org/10.21203/rs.3.rs-212095/v1

License: (c) (i) This work is licensed under a Creative Commons Attribution 4.0 International License.

Read Full License

Version of Record: A version of this preprint was published at Systematic Reviews on November 15th, 2021. See the published version at https://doi.org/10.1186/s13643-021-01853-9. 


\section{Abstract}

\section{Background}

Idiopathic pulmonary fibrosis (IPF) is one of the common types of interstitial lung disease having high prevalence and mortality worldwide. As a result of patient-centred hindering factors of adherence to centre-based pulmonary rehabilitation (PR), home-based PR is an alternate mode of rehabilitating individuals with IPF. This systematic review will evaluate the effectiveness of unsupervised home-based PR on functional capacity and health-related quality of life (HRQoL) in individuals with IPF.

\section{Methods}

Clinically stable, high resolution computed tomography and physician diagnosed IPF participants having modified Medical Research Council score below five will be considered for the systematic review. Studies involving home-based PR as an intervention to treat individuals with IPF will be considered. Randomised controlled trials and quasi-randomised studies (with two groups followed-up) are eligible to be included. Outcomes of our interest are functional capacity (6-minute walk distance, shuttle walk test and incremental shuttle walk test) and secondary outcome measure would include assessment of quality of life and adverse effects of intervention. Electronic databases such as SCOPUS, Medline (PubMed and Web of Science), PEDRo and CINAHL will be searched using database specific terms. Additionally, forward and backward citations of included studies will be searched to identify potential records. Two review authors, independently, will conduct the screening, data extraction using a customised standard tool, and critical appraisal using Cochrane Risk of Bias 2 tool of included studies. If data permits, metaanalysis will be conducted. In case of substantial heterogeneity, we will do a narrative synthesis. Subgroup analysis will be undertaken based on various contextual and interventional factors.

\section{Discussion}

This systematic review will guide the physiotherapists and individuals with IPF on the effectiveness of unsupervised home-based PR that might support and promote participation in PR for better living.

Systematic review registration

Protocol has been registered on PROSPERO CRD42020213883.

\section{Background}

'Idiopathic pulmonary fibrosis' (IPF), one of the prime variants of 'idiopathic interstitial pneumonia', is a recurrent, progressive, irreversible and generally lethal lung disease of unknown origin ${ }^{1}$. Men and women are frequently affected, with poor median survival; and the survival varies between two and five years ${ }^{1,2}$. The incidence of IPF varies across the globe and ranges between 0.2 and 93.7 per 100,000 population per year ${ }^{2}$. There is diversity in age standardised mortality rate of IPF between different countries (4.64 to 
8.28 per 100,000 population); however, data were mostly available from few high-income countries ${ }^{2}$. Increasing trend in IPF has been reported over the years in many countries ${ }^{2}$. Cigarette smoking and exposure to metal and wood emissions were the most important environmental threats for developing IPF $1,4,5$.

IPF individuals seek treatment for chronic and progressive cough and dyspnoea. Dyspnoea and fatigue deteriorate functional ability and quality of life in individuals with IPF. As fibrosis progresses, dyspnoea and fatigue intensify, individual with IPF become gradually less physically involved and unable to perform physical tasks $3,6,7$.

Pharmacotherapy (pirfenidone and nintedanib) is a promising approach in the management of mild to moderately impaired lung function tests among IPF ${ }^{8}$. Additionally, IPF involves the application of homebased pulmonary rehabilitation (PR) program to optimise functional outcomes 9,10 . Individually tailored exercise training is the cornerstone of PR. The exercise training component included both aerobic and strength training ${ }^{9,11}$ and each session may consist of up to 30 minutes of aerobic training ${ }^{12}$. PR, a systematic technique, improves dyspnoea, enhances exercise efficiency, and improves health-related quality of life (HRQoL) ${ }^{13}$. PR is commonly delivered in an outpatient or community setting and may comprise of two - three sessions per week ${ }^{14}$. Disease-specific education and self-management training helps people with IPF to develop and implement the skills necessary to perform the tasks, guide behaviour change and provide support to achieve optimal function ${ }^{14,15}$.

Despite the proven benefits of PR for individuals with 'chronic respiratory diseases' (CRD), a small percentage of individuals who are eligible to attend PR adhere to it on regular basis ${ }^{16}$. Some of the patient-centred barriers to attendance and adherence of PR are related to travel and transport to the rehabilitation centre. Lack of services and suitably trained healthcare professionals, especially in nonmetropolitan areas make it difficult to avail the services ${ }^{16,17}$. Home-based PR has the potential to overcome known barriers to PR participation and could be a relevant treatment alternative across all CRD including IPF ${ }^{14}$.

The empirical research has shown the benefits of PR in enhancing functional capability and HRQoL, thereby minimising hospitalisation and frequency of hospital stay ${ }^{18}$. PR programs are demonstrated to be successful by home-based mode of rehabilitation for training, and therefore, previous research had recommended creation of such programmes ${ }^{19,20,21}$. Home-based PR programmes, for a larger proportion of IPF individuals living in lesser developed countries or rural and remote surroundings, may contribute to broader provision of PR. Furthermore, unsupervised home-based PR can be a promising approach for a resource-limited remote area of the world.

Previous systematic reviews for supervised exercise training programs have exhibited clinical benefits in ameliorating exercise capacity, dyspnoea and quality of life in individuals with IPF 9,12,22. A systematic review ${ }^{9}$ included studies conducted a mixture of centre- and home-based setting to assess the effects of 
exercise-based PR in individuals with IPF. As the subgroup analysis was not conducted it is difficult to comment on which of the two settings proved to be beneficial. The underlying effects of chronic adaptation to a regular non-supervised home-based PR on functional capacity in IPF has yet to be described by a systematic review level evidence. There has not been a comprehensive assessment of the capacity of non-supervised home-based PR to achieve improvements in functional capacity using 6minute walk test ${ }^{23}$, shuttle walk test ${ }^{24}$, incremental shuttle walk test and HRQoL ${ }^{18,25,26}$ in people with IPF, or its ability to improve uptake and access to rehabilitation services.

The current systematic review is, therefore, planned to evaluate the effectiveness of an unsupervised selfcare home-based PR training program on the functional capacity in individuals with IPF. The evidence generated from this systematic review will guide policy makers, researchers and public health practitioners in designing evidence-based PR program performed at home, which might help in improving the HRQoL, activity of daily living and bring about functional improvements among people with IPF. It would provide basis for establishing a standard protocol and an alternative to supervised centre-based rehabilitation programs for individuals with IPF.

\section{Material And Methods}

This systematic review protocol has adhered to PRISMA-P guidelines ${ }^{27}$. The protocol is registered under PROSPERO, CRD42020213883.

\section{Inclusion and exclusion criteria}

Participants: Individuals with IPF diagnosed using high resolution computed tomography findings and by registered physician will be included. IPF could be of any severity but, the individual should be in a stable clinical state. Individuals with IPF having dyspnoea with modified Medical Research Council score of grade 5 would be excluded from the review. Studies on surgically treated lung transplanted IPF individuals will be excluded as their care and rehabilitation pathways differ significantly from those with IPF individuals without lung transplant. Studies with mixed population will be excluded however, we will include the study if it has carried out subgroup analysis of population of our interest. We will exclude other forms of interstitial lung disease other than IPF. There would be no restriction on the duration of diagnosis of IPF and the age of the population.

Intervention: The intervention eligible to be included in this review is a comprehensive home-based PR, based on exercise training. Home-based PR is a selfcare-based intervention, in which the physiotherapist/professional provides training and the participant perform the PR by themselves at home. This intervention is mostly unsupervised, but caregiver supervised PR are eligible to be included. We will include studies with physiotherapist/any professionally trained individual periodically (minimum once in 15 days) supervising the participant, either at the participant's home, community or hospital setup. This supervision could be web- or tele-based. Home-based PR may be performed in a group or individually in the community. To be included, studies must consider home-based PR programmes that 
have a component of aerobic exercise, resistance exercise, or both, with or without health education. Nevertheless, education programs without home-based PR will be excluded. Minimum intervention duration should be four weeks but, could be of any frequency per week. We will exclude studies that provided single exercise programs. If the first and single training PR session has been delivered at centre/hospital, but remaining sessions were carried out at home/community setup, we will include the study. We will consider studies with individuals undergoing pharmacotherapy or any other standard care, but the co-intervention should have been equally distributed in both the groups. If the study included mixture of home-based and centre-based PR, we would include it provided there is subgroup available for unsupervised home-based PR.

Comparisons: Conventional supervised, centre-based PR treatment, no treatment or standard care. We will include studies that compare unsupervised home-based PR with traditional/ conventional PR or no rehabilitation. Comparison could be drawn with centre-based PR, or between the providers/ supervisors. Within home-based PR, comparison could be between two different forms (e.g., aerobic home-based PR compared to strength training home-based PR compared to strength training home-based PR), duration or intensity.

Outcomes: Primary outcome measures are functional capacity, as measured by 6-minute walk distance, shuttle walk test, or incremental shuttle walk test. Secondary outcome measures are condition specific quality of life measured using scales such as St. George respiratory questionnaire ${ }^{26}$, Chronic Respiratory Distress Questionnaire ${ }^{17}$ and King's Brief Interstitial Lung Disease Questionnaire ${ }^{28,29}$. We will exclude other generic tools for assessing quality of life such as WHO BREF and SF-36 (Short form-36). We will also measure side-effects of PR such as fatigue and muscle weakness, but not limited to, desaturation and exacerbation of dyspnoea.

Study type: Randomised controlled trials (RCTs) with a parallel, cluster or cross-over design and quasirandomised studies with at least two groups (intervention and control), followed-up for at least four-week duration will be included. Non-randomised studies (with single group), observational studies, letters to editor and reviews will be excluded.

\section{Conceptual framework of the current systematic review}

We hypothesise the relationship between the effects of home-based PR on individuals with IPF, the associated determinants and its resultant effects on the outcomes through the conceptual framework (Fig. 1). Several determinants (involving factors related to the individual, family, and healthcare) have a direct or indirect influence on the overall well-being of the IPF individuals and the intervention (homebased PR). Considering the poor prognosis and patient-centred barriers, it is anticipated that home-based PR will bring about improvements in the functional outcomes and quality of life of individuals with IPF.

\section{Study methods:}


Electronic searches: Medline (via PubMed and Web of Science), SCOPUS, CINHAL and PEDRo will be searched for English publications until January 31, 2021. Preliminary search strategy will be developed in Medline (PubMed) and will be customised for other databases. There will not be restriction on publication status of the articles.

Searching other resources: To identify in progress and unpublished studies, 'Clinical Trials registry of India', and 'the WHO International Clinical Trials Registry platform' will be scanned. The backward and forward citations of included studies will be scanned to look for any potentially eligible records. We will use Rayyan software for managing the data and screening the records.

Keywords: Functional capacity; Home-based pulmonary rehabilitation; Health-related quality of life; Idiopathic pulmonary fibrosis

\section{Data extraction and analysis.}

Study selection: Two review authors (RA and VK) will independently review titles and abstracts retrieved from the search and identify all potentially eligible studies. Full text of included studies will be obtained, and the same team members will review independently according to the inclusion criteria. We will address disputes, if any, through discussion before consensus, and have a third review author (AM or SSP) for final decision when consensus cannot be achieved. The review authors shall document the rationale for excluding all full texts which do not meet the criteria for inclusion. The study selection process will be documented using PRISMA flow diagram.

Data extraction: We will customise and use Cochrane EPOC data collection form ${ }^{30}$ for extracting relevant information from included studies, which will be piloted on at least two studies. From each included study, we will extract information on title, year of publication, author, study design, type of analysis, number of included participants, country, population (e.g., age, gender and other contextual information), severity of IPF, comorbidity, intervention (type, duration, intensity, frequency, provider etc), comparison and outcome (follow-up, tool used, outcome assessor etc). Corresponding authors of the included studies will be contacted in case of limited or lack of information in the studies. However, studies will be excluded if we do not receive a reply from the corresponding author within a fortnight.

Critical appraisal of included studies: RA and VK, independently, will assess methodological quality of included studies using 'Cochrane Risk of Bias $2^{\prime}$ tool ${ }^{31}$. Both the reviewers will clear the dissent, if any, by discussion. ROB 2 tool will assess bias at selection, blinding of participants, outcome assessment, reporting and other bias. We will judge each possible source of bias as high, low or some concern; and provide a quote from the study report along with a justification. We will overcome disagreements by discussion until consensus for our decision on the 'Risk of bias'.

Data synthesis: Studies will be examined for methodological, clinical and statistical heterogeneity. The statistical heterogeneity between trials will be evaluated using I square or Chi square statistics. The values will be categorised as 'no' (<25\%), 'low' (25-49\%), 'moderate' (50-75\%) or 'substantial' (>75\%) 
heterogeneity. Depending on the statistical heterogeneity, we will apply fixed or random effects model. Using RevMan, if possible, we will individually combine studies into a single meta-analysis using the generic method of inverse variance and will estimate the treatment effects reported by individual studies. Effect estimates such as odds ratios or risk ratios (for categorical data) and weighted/ standardised mean differences (for continuous data) and their $95 \%$ confidence intervals will be computed. In case of substantial statistical heterogeneity, or methodological and clinical heterogeneity we will undertake narrative synthesis and subgroup analysis. Subgroups could be based on severity of IPF (or clinical variables), age, other contextual factors, and study designs.

\section{Discussion}

IPF is a chronic, debilitating and fatal disease among other ILD's ${ }^{1}$. PR has known effects on functional capacity and quality of life in a supervised centre-based setup for individuals with IPF 9,12,22. The current review will provide insights regarding the effectiveness of home-based PR on functional capacity and HRQoL in individuals with IPF. This systematic review will guide the physiotherapists and individuals with IPF on the effectiveness of home-based PR that might support and promote participation in PR for better living.

To our best of knowledge this would be the first systematic review to comprehensively assess the effectiveness of unsupervised home-based PR on functional capacity and quality of life in individuals with IPF. Due to restrictions in accessing the databases, search would be limited to a few databases for this systematic review. Due to resource limitations, non-English publications will not be included. We anticipate variability in terms of population and the way PR is performed in different regions, which may hinder pooling the result. To mitigate this issue, we have planned subgroup analysis. Considering the intervention defined in the review protocol, it can be anticipated that some studies might not clearly report whether the participants have been trained or the exercise training was supervised. We will contact the corresponding authors if there is limited or lack of information.

\section{Abbreviations}

CRD-Chronic Respiratory Disease; EPOC- Effective Practice and Organisation of Care; HRQoL- Health Related Quality of Life; IPF- Idiopathic Pulmonary Fibrosis; ILD- Interstitial Lung Disease; PR- Pulmonary Rehabilitation; PROSPERO- The International Prospective Register of Systematic Reviews; PRISMA PPreferred Reporting Items for Systematic Review and Meta-analysis; PEDRo- The Physiotherapy Evidence Database; SF 36- Short Form 36; WHO BREF- The World Health Organization Quality of Life Instrument.

\section{Declarations}

Ethics approval and consent to participate: Not applicable

Consent for publication: Not applicable 
Availability of data and materials: Data sharing is not applicable to this protocol as datasets are not generated or analysed.

Competing interests: The authors declare that they have no competing interests.

Funding: No funding involved.

Author contributions: VK, AM and RA were involved in conception of the systematic review. VK, RA and SSP were involved in designing and drafting the protocol. AM, AKM, SSP and UN were involved in substantially revising the systematic review protocol. VK is the guarantor of the review.

Acknowledgement: The authors would like to acknowledge Manipal Academy of Higher Education, MAHE, for providing them with technical support required for the study.

\section{References}

1. Raghu G, Collard HR, Egan JJ, Martinez FJ, Behr J, Brown KK, et al. An official ATS/ERS/JRS/ALAT statement: idiopathic pulmonary fibrosis: evidence-based guidelines for diagnosis and management. Am J Respir Crit Care Med. 2011;183(6):788-824. doi: 10.1164/rccm.2009-040GL.

2. Hutchinson J, Fogarty A, Hubbard R, McKeever T. Global incidence and mortality of idiopathic pulmonary fibrosis: a systematic review. Eur Respir J. 2015;46(3):795-806. doi:

10.1183/09031936.00185114.

3. Cox IA, Arriagada NB, de Graaff B, Corte TJ, Glaspole I, Lartey S, et al. Health-related quality of life of patients with idiopathic pulmonary fibrosis: a systematic review and meta-analysis. European Respiratory Review 2020;29(158): 200154. doi: 10.1183/16000617.0154-2020

4. Sesé L, Nunes H, Cottin V, Sanyal S, Didier M, Carton Z, et al. Role of atmospheric pollution on the natural history of idiopathic pulmonary fibrosis. Thorax 2018;73(2):145-50. doi: 10.1136/thoraxjnl2017-209967.

5. Abramson MJ, Murambadoro T, Alif SM, Benke GP, Dharmage SC, Glaspole I, et al. Occupational and environmental risk factors for idiopathic pulmonary fibrosis in Australia: case-control study. Thorax 2020;75(10):864-9. doi: 1136/thoraxjnl-2019-214478.

6. Maheshwari U, Gupta D, Aggarwal AN, Jindal SK. Spectrum and diagnosis of idiopathic pulmonary fibrosis. Indian J Chest Dis Allied Sci. 2004;46(1):23-6.

7. van Jaarsveld MF, Gwyther L. A systematic review of literature to evaluate the burden of physical and psychological symptoms and palliative care in patients diagnosed with idiopathic pulmonary fibrosis. African Journal of Thoracic and Critical Care Medicine 2019;25(1):22-7. doi: 10.7196/sarj.2019.v25i1.231.

8. Raghu G. Pharmacotherapy for idiopathic pulmonary fibrosis: current landscape and future potential. Eur Respir Rev. 2017;26(145):170071. doi: 10.1183/16000617.0071-2017. 
9. Hanada M, Kasawara KT, Mathur S, Rozenberg D, Kozu R, Hassan SA, Reid WD. Aerobic and breathing exercises improve dyspnea, exercise capacity and quality of life in idiopathic pulmonary fibrosis patients: systematic review and meta-analysis. J Thorac Dis. 2020;12(3):1041. doi: 10.21037/jtd.2019.12.27.

10. Pleasants R, Tighe RM. Management of idiopathic pulmonary fibrosis. Annals of Pharmacotherapy 2019;53(12):1238-48. doi: 10.1177/1060028019862497.

11. Ries AL. Pulmonary rehabilitation: summary of an evidence-based guideline. Respir Care. 2008;53(9):1203-7.

12. Yu X, Li X, Wang L, Liu R, Xie Y, Li S, Li J. Pulmonary rehabilitation for exercise tolerance and quality of life in IPF patients: a systematic review and meta-analysis. BioMed Res. Int. 2019;2019. doi: 10.1155/2019/8498603.

13. Halding AG, Heggdal K. Patients' experiences of health transitions in pulmonary rehabilitation. Nurs. Inq. 2012;19(4):345-56. doi: 10.1111/j.1440-1800.2011.00573.x.

14. Rochester CL, Vogiatzis I, Holland AE, Lareau SC, Marciniuk DD, Puhan MA, et al. An official American Thoracic Society/European Respiratory Society policy statement: enhancing implementation, use, and delivery of pulmonary rehabilitation. Am J Respir Crit Care Med. 2015;192(11):1373-86. doi: 10.1164/rccm.201510-1966ST.

15. Lee JY, Tikellis G, Corte TJ, Goh NS, Keir GJ, Spencer L, Sandford D, Khor YH, Glaspole I, Price J, HeyCunningham AJ. The supportive care needs of people living with pulmonary fibrosis and their caregivers: a systematic review. Eur Respir Rev. 2020;29(156). doi: 10.1183/16000617.0125-2019.

16. Cox NS, McDonald CF, Alison JA, Mahal A, Wootton R, Hill CJ, et al. Telerehabilitation versus traditional centre-based pulmonary rehabilitation for people with chronic respiratory disease: protocol for a randomised controlled trial. BMC Pulmonary Medicine 2018;18(1): 71. doi: 10.1186/s12890-018-0646-0.

17. Holland AE, Hill CJ, Conron M, Munro P, McDonald CF. Short term improvement in exercise capacity and symptoms following exercise training in interstitial lung disease. Thorax 2008;63(6):549-54. doi: 10.1136/thx.2007.088070.

18. Huppmann P, Sczepanski B, Boensch M, Winterkamp S, Schönheit-Kenn U, Neurohr C, et al. Effects of inpatient pulmonary rehabilitation in patients with interstitial lung disease. European Respiratory Journal 2013;42(2):444-53. doi: 10.1183/09031936.00081512.

19. Holland AE, Mahal A, Hill CJ, Lee AL, Burge AT, Moore R, et al. Benefits and costs of home-based pulmonary rehabilitation in chronic obstructive pulmonary disease-a multi-centre randomised controlled equivalence trial. BMC Pulmonary Medicine 2013;13(1):57. doi: 1186/1471-2466-13-57.

20. Marciniuk DD, Brooks D, Butcher S, Debigare R, Dechman G, Ford G, et al. Optimizing pulmonary rehabilitation in chronic obstructive pulmonary disease-practical issues: a Canadian Thoracic Society Clinical Practice Guideline. Can Respir J. 2010;17:159-68. doi: 10.1155/2010/425975.

21. Vasilopoulou M, Papaioannou Al, Kaltsakas G, Louvaris Z, Chynkiamis N, Spetsioti S, et al. Homebased maintenance tele-rehabilitation reduces the risk for acute exacerbations of COPD, 
hospitalisations and emergency department visits. Eur Respir J. 2017;49(5): 1602129. doi: 10.1183/13993003.02129-2016.

22. Gomes-Neto M, Silva CM, Ezequiel D, Conceição CS, Saquetto M, Machado AS. Impact of pulmonary rehabilitation on exercise tolerance and quality of life in patients with idiopathic pulmonary fibrosis: a systematic review and meta-analysis. J Cardiopulm Rehabil Prev. 2018;38(5):273-8. doi: 1097/HCR.0000000000000273.

23. Ozalevli S, Karaali HK, Ilgin D, Ucan ES. Effect of home-based pulmonary rehabilitation in patients with idiopathic pulmonary fibrosis. Multidisciplinary Respiratory Medicine 2010;5(1):31. doi: 1186/2049-6958-5-1-31.

24. Moloney ED, Clayton N, Mukherjee DK, Gallagher CG, Egan JJ. The shuttle walk exercise test in idiopathic pulmonary fibrosis. Respiratory medicine 2003;97(6):682-7. doi: 10.1053/rmed.2003.1501.

25. Nolan CM, Delogu V, Maddocks M, Patel S, Barker RE, Jones SE, et al. Validity, responsiveness and minimum clinically important difference of the incremental shuttle walk in idiopathic pulmonary fibrosis: a prospective study. Thorax 2018;73(7):680-2. doi: 1136/thoraxjnl-2017-210589

26. Swigris JJ, Esser D, Conoscenti CS, Brown KK. The psychometric properties of the St George's Respiratory Questionnaire (SGRQ) in patients with idiopathic pulmonary fibrosis: a literature review. Health Qual Life Outcomes. 2014;12(1):124. doi: 10.1183/13993003.01788-2016.

27. Moher D, Shamseer L, Clarke M, Ghersi D, Liberati A, Petticrew M, et al. Preferred reporting items for systematic review and meta-analysis protocols (PRISMA-P) 2015 statement. Systematic reviews 2015;4(1):1. doi: 10.1186/2046-4053-4-1.

28. Nolan CM, Birring SS, Maddocks M, Maher TM, Patel S, Barker RE, et. al. King's Brief Interstitial Lung Disease questionnaire: responsiveness and minimum clinically important difference. Eur Respir $\mathrm{J}$. 2019;54(3): 1900281. doi: 10.1183/13993003.00281-2019

29. Olson AL, Brown KK, Swigris JJ. Understanding and optimizing health-related quality of life and physical functional capacity in idiopathic pulmonary fibrosis. Patient Relat Outcome Meas. 2016;7:29. doi: 2147/prom.s74857.

30. Cochrane Effective Practice and Organisation of Care (EPOC). Data collection form. EPOC Resources for review author 2017. Available from: https://epoc.cochrane.org/resources/epoc-specific-resourcesreview-authors

31. Sterne JA, Savović J, Page MJ, Elbers RG, Blencowe NS, Boutron I, et al. RoB 2: a revised tool for assessing risk of bias in randomised trials. The BMJ. 2019;366. Doi: 10.1136/bmj.I4898.

\section{Figures}




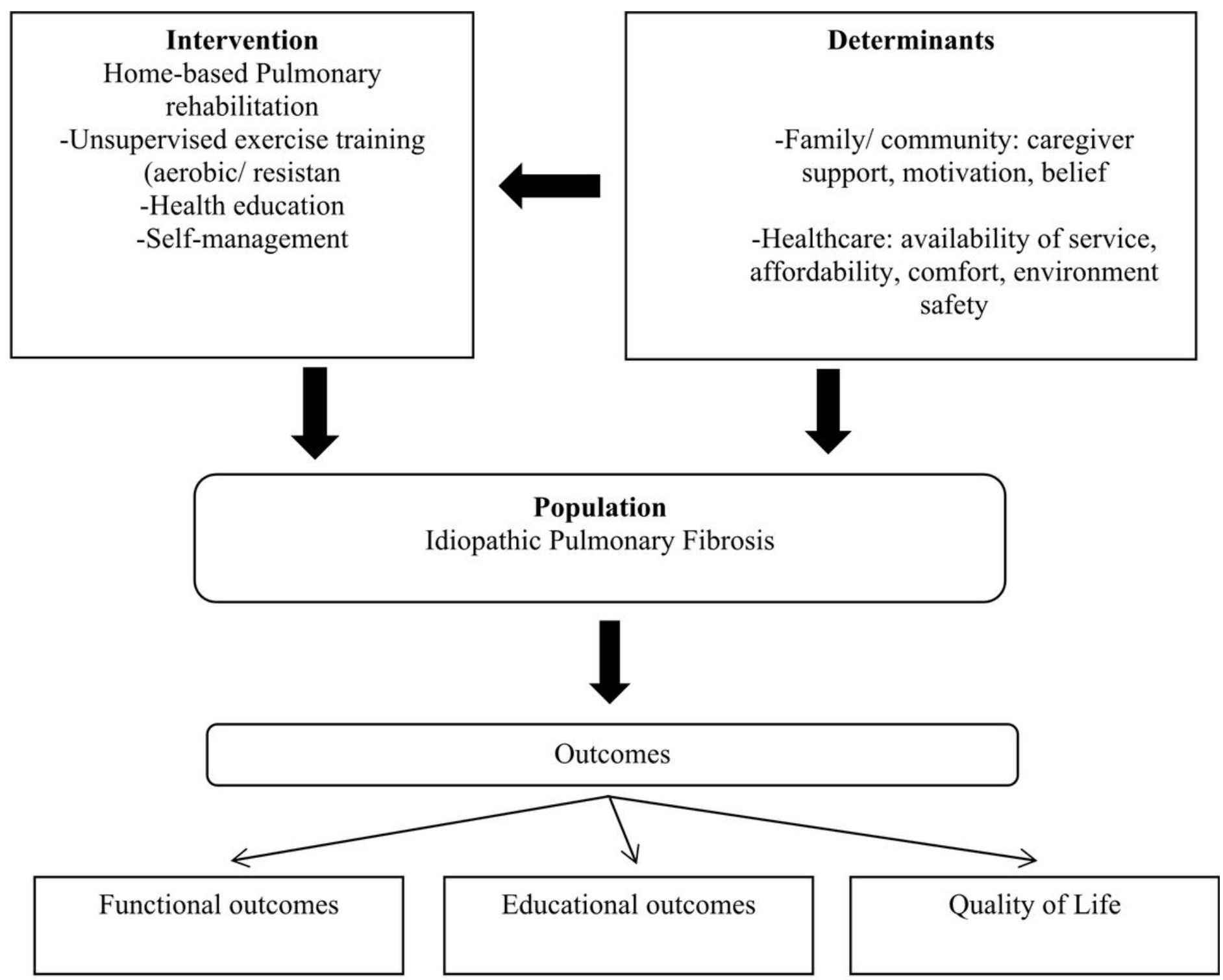

\section{Figure 1}

Conceptual framework for the effects of home-based pulmonary rehabilitation on individuals with idiopathic pulmonary fibrosis. 